\title{
Topographic mapping of a specimen after endoscopic submucosal dissection
}

\section{다)(1) $\odot$}

\author{
Authors \\ Alanna Ebigbo ${ }^{1}$, Andreas Probst ${ }^{1}$, Helmut Messmann ${ }^{1}$, Bruno MärkI², Yun-Chung Nam-Apostolopoulos ${ }^{2}$ \\ Institutions \\ 1 Department of Gastroenterology, Klinikum Augsburg, \\ Germany \\ 2 Institute of Pathology, Klinikum Augsburg, Germany \\ submitted 18.11 .2018 \\ accepted after revision $\quad 27.12 .2018$ \\ Bibliography \\ DOI https://doi.org/10.1055/a-0846-2043 | \\ Endoscopy International Open 2019; 07: E521-E524 \\ (c) Georg Thieme Verlag KG Stuttgart · New York \\ ISSN 2364-3722 \\ Corresponding author \\ Dr. Alanna Ebigbo, Klinikum Augsburg, Stenglinstr. 2, \\ 86156 Augsburg, Germany \\ Fax: +00498214002748 \\ Alanna.ebigbo@klinikum-augsburg.de

\section{ABSTRACT} \\ Background and study aims Mapping of pathologic spe- \\ cimens after endoscopic submucosal dissection (ESD) is \\ common practice in Asian countries, especially in Japan. \\ However, there is a lack of awareness for this technique in \\ Europe. In this report, we demonstrate the feasibility and \\ benefits of topographic mapping in a Western setting.
}

\section{Introduction}

Endoscopic submucosal dissection (ESD) is an excellent instrument for en bloc resection of precancerous and early cancerous lesions in the gastrointestinal tract but with a steep learning curve $[1,2]$. For successful en bloc ESD, a thorough diagnostic evaluation of the lesion should be done prior to endoscopic resection [3]. However, this can be challenging even with use of endoscopic classification methods such as Kudo's pit-pattern or the Japanese NBI expert team (JNET) classification [4].

Feeding information back to endoscopists after histopathological assessment could improve their diagnostic ability and, in total, improve the quality of ESD. This method of "mapping and virtual reconstruction" involves close collaboration between an endoscopist and a pathologist. In countries like Korea and Japan, it is implemented routinely for pathological work-up of ESD specimens, and correlates all conspicuous features observed in the endoscopic image with its corresponding histopathology [5].

In this report, we demonstrate the principle of mapping and virtual reconstruction after ESD resection of a large colonic adenoma, which had a small region of shallow submucosal cancer.

\section{Methods}

A laterally spreading tumor (LST) of about $40 \mathrm{~mm}$ diameter in the proximal rectum was examined extensively prior to ESD resection. The surface and vascular pattern of the lesion was described using Sano's classification as well as the JNET classification $[6,7]$. Endoscopic examination of the lesion was done with an Olympus GIF-190 series endoscope using white light, narrow-band imaging (NBI), indigocarmine-chromoendoscopy (IC) and near-focus magnification.

After ESD resection, the specimen was stretched and pinned at its outer borders onto a cork plate using standard office pins. A macroscopic close-up image of the specimen was taken using a standard digital camera. The specimen was fixed in formalin for about 24 hours after which a second macroscopic image was taken. A third photographic image was taken after cutting and sectioning of the specimen. Each section was numbered to make mapping and reconstruction possible. Finally, the histopathology of each section was projected onto the macroscopic image. Most importantly, suspicious regions or areas with interesting features seen on endoscopy were correlated with the histology. Lines of different colors were used to designate the extent of normal tissue, tissue with low-grade dysplasia, highgrade dysplasia and cancer. 

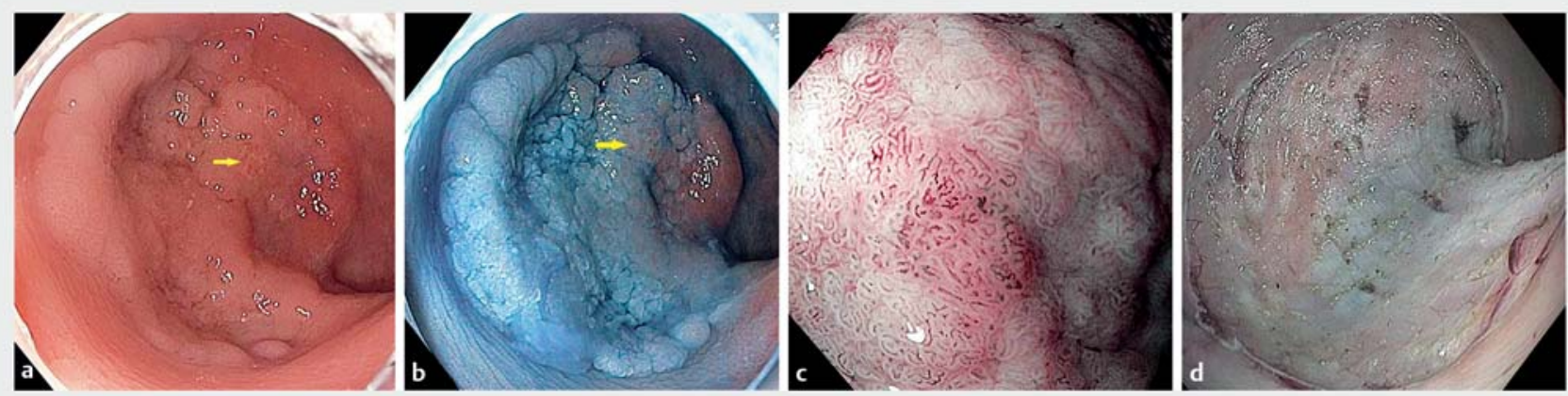

- Fig. 1 a White light. b IC-Staining. c NBI magnified view (Sano IIla/JNET 2B). d ESD resection.
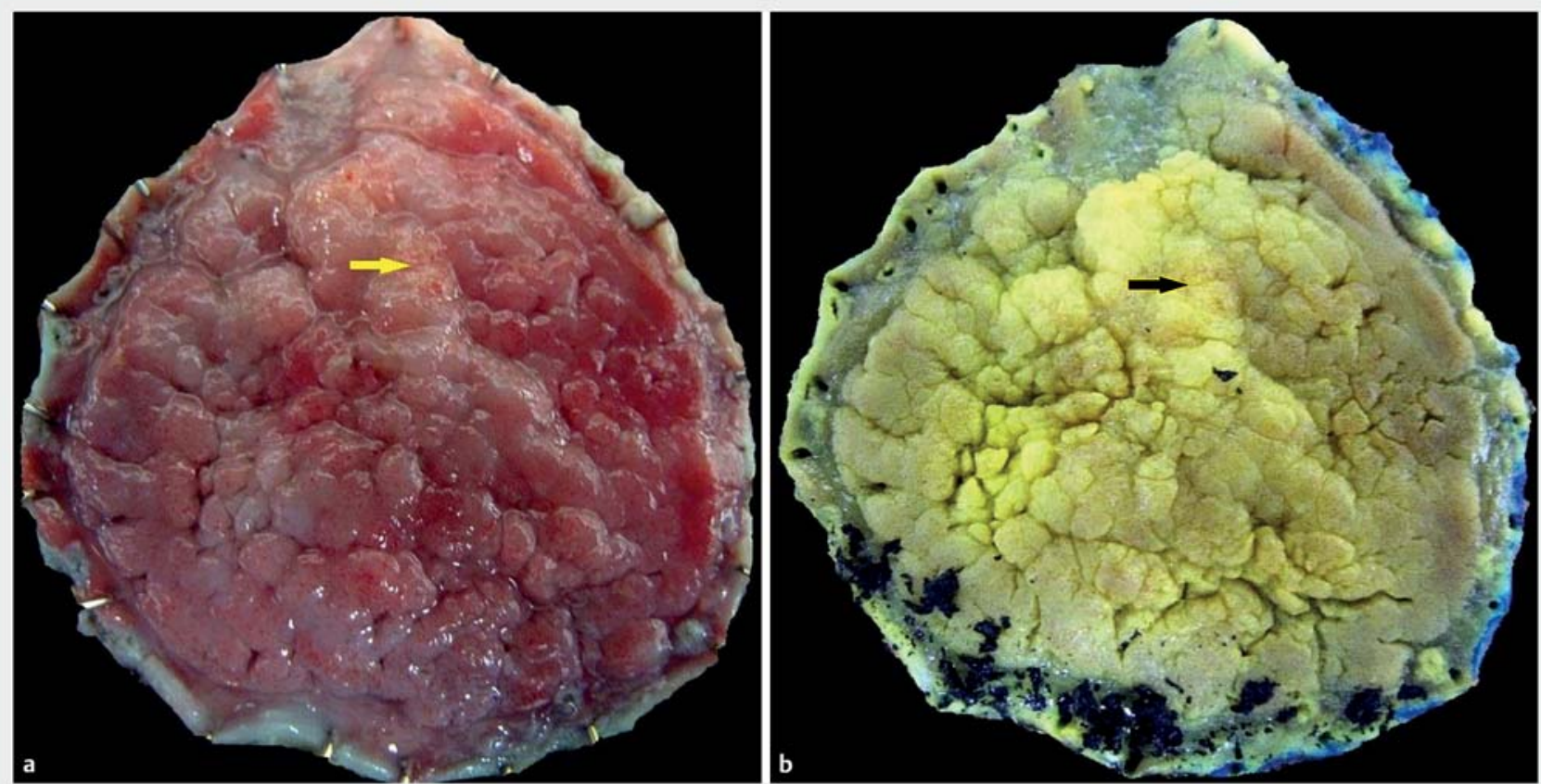

Fig. 2 a Macroscopic image before fixation. b Macroscopic image after fixation. The upper part of the image is the oral side while the lower part is the anal side of the lesion.

\section{Results}

The endoscopic images of the laterally spreading tumor with a diameter of about $40 \mathrm{~mm}$ in the rectum are shown in $\mathbf{F i g . 1 a}$ and $>$ Fig. 1b. A greater part of the tumor has a granular surface type but a small area at the right side with a non-granular surface is also present. This non-granular area remained reddish after IC staining.

A small region (yellow arrow) with an irregular surface was evaluated more closely using the magnified NBI mode ( $\triangleright$ Fig. 1c). Here an irregular vascular pattern and diminished vessel density can be seen ( $\nabla$ Fig. 1c). This area can be classified as Sano Type IIIA and JNET Type 2B. > Fig. 1d shows the appearance after ESD.

The macroscopic images of the specimen before and after formalin fixation as well as sectioning are shown in $>$ Fig. 2 a and $>$ Fig. 2b. Again, the yellow arrow points at the suspicious region described above. In $>$ Fig. 3 a the image of the sectioned specimen is shown. Sections are numbered from top to bottom to make identification possible. Each section is examined histologically after which the histologic results are transferred onto the macroscopic images. This process, called mapping, results in an image with lines of different color depicting the various histologic patterns ( $\mathbf{F i g . 3 b}$ ).

In > Fig. 4a, $>$ Fig. 4b, > Fig.4c, and $>$ Fig. 4d, the histopathology of section number 4 (counting from the top of the image) is demonstrated. It now becomes clear that the area with a granular type surface showed high-grade intraepithelial neoplasia while the non-granular type region had low-grade dysplasia. Histology of the small suspicious area with Sano class IIIA and JNET Type 2B pattern showed a shallow submucosal invasive cancer (pT1 G2 $600 \mu \mathrm{m}$ ). 

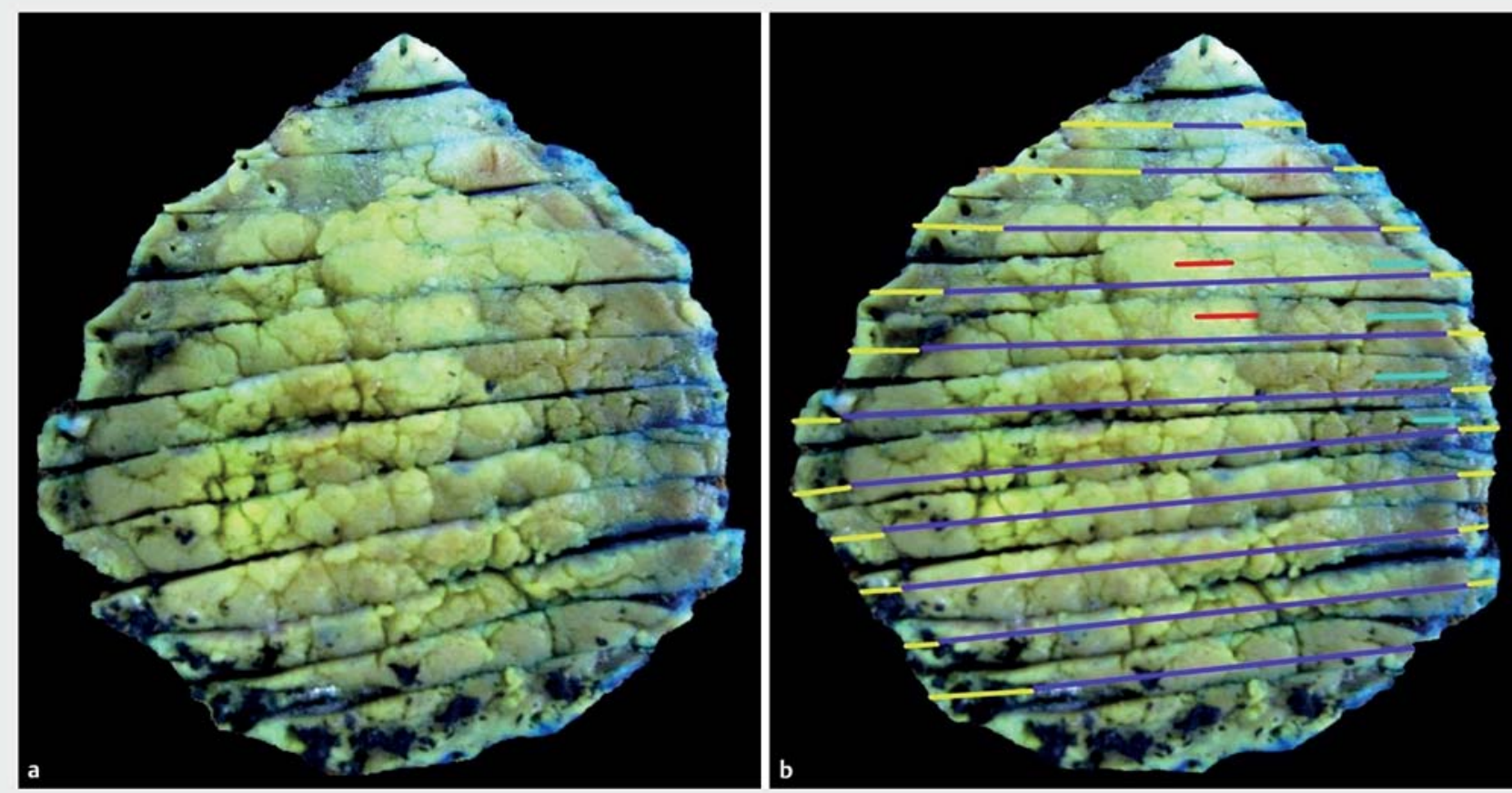

Fig. 3 a Macroscopic image after sectioning. b Macroscopic image after mapping. The upper part of the image is the oral side while the lower part is the anal side of the lesion. The red lines show the area of pT1 adenocarcinoma.
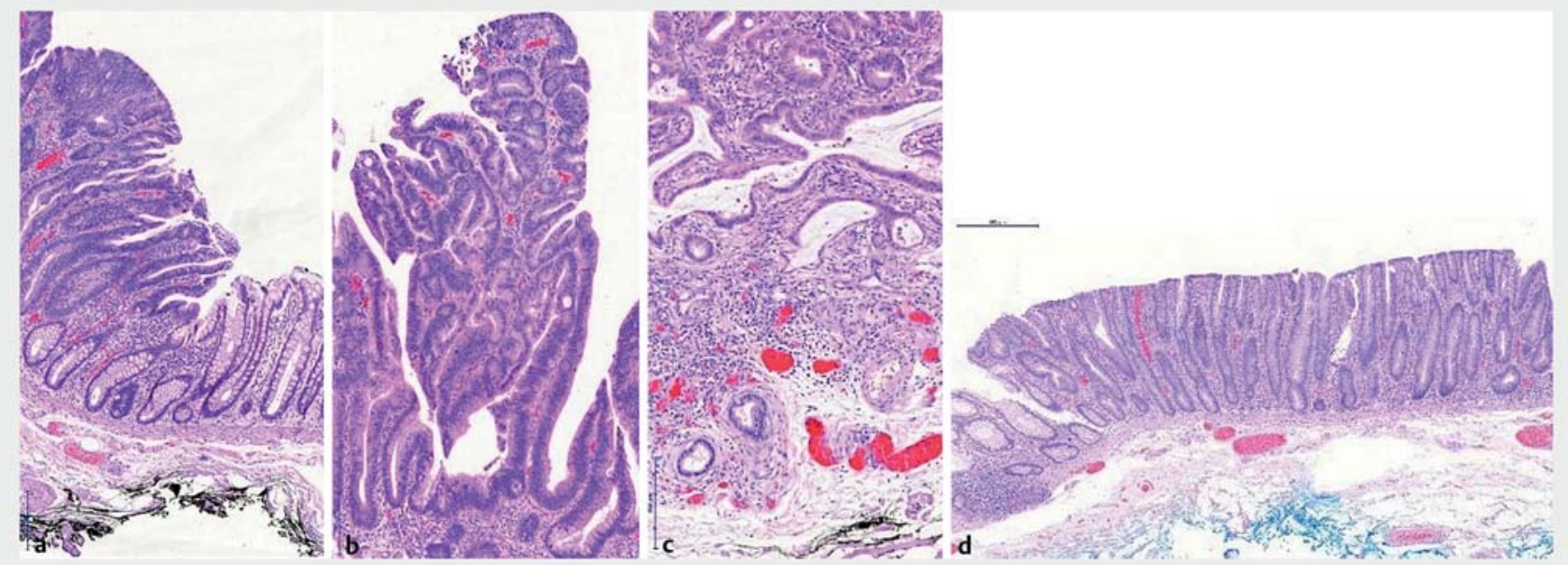

- Fig. 4 a Transition from normal mucosa to tubulo-villous adenoma - depicted as the yellow line (normal mucosa) and the dark blue line (tubulo-villous adenoma) in $>$ Fig. $\mathbf{3 b}$. b Tubular adenoma with high grade intraepithelial neoplasia - dark blue line in $>$ Fig. $\mathbf{3 b}$. c Adenocarcinoma pT1, G2, sm2 $(600 \mu \mathrm{m})$ - depicted as the red line in Fig. 3b. d Tubular adenoma with low grade intraepithelial neoplasia - depicted as the neon blue line in $>$ Fig. $\mathbf{3 b}$.

\section{Discussion}

In this histopathological work-up of an ESD specimen from a rectal LST with a small region of superficial submucosal cancer, we demonstrated that the process of mapping leads to excellent topographical reconstruction of the lesion and the specimen. An exact correlation of the histopathology with all subtle features observed during the endoscopic examination was possible. The lesion shown in this report was chosen deliberately to demonstrate the principles and benefits of reconstruction and mapping. Ordinarily, the pathologic report in this case would not have provided an exact correlation with the various features observed during endoscopy.

Sometimes, it may be difficult to compare the endoscopic view to the corresponding region of interest on the specimen. This problem can be easily solved by pressing two surface marks with an argon-plasma-coagulation (APC) probe at both 
sides of the region of interest, making it more visible for the pathologist. Furthermore, an APC mark can be placed at the proximal end of the lesion, which helps orientate the specimen after en bloc resection.

Mapping of ESD specimens is not yet common practice in the West [8]. Detailed mapping of specimens demonstrated in this paper goes further than the pathologic workup described by Kumarasinghe et al [9] in which a standardized protocol for handling, grossing, and assessing specimens was proposed. However, it does not include the detailed mapping demonstrated in our paper. Although the advantages of this mapping process are obvious, one limitation may be time constraints for endoscopists and pathologists, which could hinder its implementation in daily routine practice. Also, it involves a close discussion between the pathologist and the endoscopist, which may not always be possible or practical in a daily routine. A compromise for the European setting could be to reduce the mapping and reconstruction only to those regions of interest that were particularly conspicuous during endoscopic evaluation or at least to those regions that had a cancerous histopathology.

\section{Conclusion}

If performed routinely, mapping could improve the diagnostic ability of endoscopists because all macroscopic details can be correlated to their histology [8]. Large ESD specimens may have only a small region of cancer or even multifocal regions of cancer, such as in Barrett's specimens, which may not be identified during endoscopy. After mapping, these regions can be correlated not only onto the image of the specimen but also onto the original endoscopic image. This correlation will educate and train endoscopists, and could lead to better pretherapeutic evaluation of lesions prior to endoscopic resection.
Competing interests

None

References

[1] Oka S, Tanaka S, Kaneko I et al. Advantage of endoscopic submucosal dissection compared with EMR for early gastric cancer. Gastrointest Endosc 2006; 64: $877-883$

[2] Kotzev Al, Yang D, Draganov PV. How to master endoscopic submucosal dissection in the USA. Dig Endosc 2018: doi:10.1111/ den.13240 [Epub ahead of print]

[3] Choi J, Kim SG, Im JP et al. Endoscopic prediction of tumor invasion depth in early gastric cancer. Gastrointest Endosc 2011; 73: 917- 27

[4] Sumimoto K, Tanaka S, Shigita K et al. Diagnostic performance of Japan NBI Expert Team classification for differentiation among noninvasive, superficially invasive, and deeply invasive colorectal neoplasia. Gastrointest Endosc 2017; 86: 700 - 709

[5] Ono H, Yao K, Fujishiro M et al. Guidelines for endoscopic submucosal dissection and endoscopic mucosal resection for early gastric cancer. Dig Endosc 2016; $28: 3-15$

[6] Uraoka T, Saito $\mathrm{Y}$, Ikematsu $\mathrm{H}$ et al. Sano's capillary pattern classification for narrow-band imaging of early colorectal lesions. Dig Endosc 2011; 23: (Suppl. 01): 112-115

[7] Sumimoto K, Tanaka S, Shigita $K$ et al. Clinical impact and characteristics of the narrow-band imaging magnifying endoscopic classification of colorectal tumors proposed by the Japan NBI Expert Team. Gastrointest Endosc 2017; 85: 816-821

[8] Amirkaveh M, Tadakazu S. Proper pathologic preparation and assessment of endoscopic mucosal resection and endoscopic submucosal dissection specimens. Tech Gastrointest Endosc 2011; 13: 95-99

[9] Kumarasinghe MP, Brown I, Raftopoulos $S$ et al. Standardised reporting protocol for endoscopic resection for Barrett oesophagus associated neoplasia: expert consensus recommendations. Pathology 2014; 46: 473-480 